\title{
Using MATLAB with the Psychophysics Toolbox to present the heterochromatic fusion nystagmus stimulus
}

\author{
CHARLES A. COLLIN \\ Dalhousie University, Halifax, Nova Scotia, Canada \\ and \\ AVI CHAUDHURI \\ McGill University, Montreal, Quebec, Canada
}

\begin{abstract}
We present a program for MATLAB that generates and presents the heterochromatic fusion nystagmus stimulus. This stimulus allows assessment of isoluminant states through recordings of reflexive eye movements (optokinetic nystagmus). The reflexive nature of the subject's response makes this stimulus especially useful with nonverbal subjects, such as children and animals. Unfortunately, the stimulus is complex and difficult to program. By presenting the present program, we hope to help those who wish to use this tool in their research. The code of the function can be downloaded at www. dal.ca/ mcmullen/downloads.html.
\end{abstract}

Vision researchers often need to determine the radiance values at which two visual stimuli of different hues appear isoluminant (i.e., are of the same subjective brightness). These values vary in complex ways with the spectral energy profile of the radiant. Also, the exact values vary across individual subjects and can vary across time in the same subject. For this reason, isoluminance points are typically determined empirically for each subject and may have to be measured repeatedly during the course of an experiment. This being the case, it is advantageous to be able to assess the isoluminant point as quickly as possible.

There are a number of methods for equating the luminance of two colors, including direct comparison, minimum border, minimum flicker, and minimum motion techniques. The interested reader is referred to Kaiser (1996) for a discussion of many of these methods and to Cavanagh and colleagues (Cavanagh, Anstis, \& Mather, 1984; Cavanagh, Tyler, \& Favreau, 1984) regarding the minimum motion technique (MMT). Chaudhuri and colleagues (Chaudhuri \& Albright, 1990, 1992; Collin \& Chaudhuri, 1996, 1998) have developed another technique for luminance matching called heterochromaticfusion nystagmus (HFN). HFN has a number of advantages that could make it the optimal stimulus for isoluminance measures in many situations. For instance, as the name implies, the HFN pattern generates optokinetic nystagmus $(\mathrm{OKN})$. OKN is a form of reflexive eye movement observed when subjects are presented with a panning visual field, as when one looks at

This work was supported by a grant from the Fonds pour la Formation de Chercheurs et l' Aide à la Recherche to C.A.C. Correspondence concerning this article should be addressed to C. A. Collin, National Research Council, 1200 Montreal Road, M-36, \#1115, Ottawa, ON K1A 0RG Canada (e-mail: charles.collin@nrc.ca). passing terrain from a moving vehicle. These eye movements can be recorded by an eye tracking system such as Eyelink II (SR Research, Mississauga, Ontario; www.eyelinkinfo.com). The Eyelink Toolbox (Cornelissen, Peters, \& Palmer, 2002) can be used to interface between MATLAB and Eyelink II. Those interested in more detail about how to measure OKN are referred to Chaudhuri and Albright (1990, 1992).

Because OKN is reflexive it provides an advantageous way to indirectly measure the responses of subjects to visual stimuli. This is especially the case with nonverbal subjects, such as young children and animals. In the case of the HFN stimulus, a subject's OKN eye movements are maximized when the stimulus is isoluminant. The eye movements minimize and then reverse direction as the stimulus diverges from isoluminance. Thus, the HFN stimulus provides a positive end point measurement. That is, a maximization in the quantity of interest (subjective isoluminance) occurs simultaneously with a maximum in the observed quantity ( $\mathrm{OKN}$ eye movements). For this reason, the term maximum motion technique has also been used to refer to this technique (see Collin \& Chaudhuri, 1998).

The fact that it provides a positive endpoint measurement gives the HFN stimulus an advantage over some similar techniques, such as MMT (Cavanagh, Anstis, \& Mather, 1984; Cavanagh, Tyler, \& Favreau, 1984) and preferentiallooking paradigms (Kaiser, 1996) in which the signal being measured is minimized in association with the achievement of isoluminance. The advantage arises from the fact that a positive measure is less subject to confusion with nonresponse and other forms of noise. For instance, the MMT results in a minimization of OKN when isoluminance is achieved. But this minimization of behavior could be confused with simple inattention or any number of 
other conditions that might cause the subject not to respond to the stimulus. This is not the case with a maximization of a structured signal (e.g., OKN), which the HFN stimulus produces.

HFN has been used to measure isoluminance points in humans and monkeys (Chaudhuri \& Albright, 1990, 1992) and to differentiate between certain types of coloranomalous vision in humans (Collin \& Chaudhuri, 1996, 1998). But its use in research has most likely been limited by the difficulty of programming the stimulus. Indeed, the stimulus is difficult to describe verbally. In the following, we first present a brief description of the stimulus and then describe how it can be produced by using MATLAB (The Mathworks Inc., Natick, MA, www.mathworks.com).

The code for a MATLAB script (HFN.m) that displays the HFN stimulus is provided in the Appendix. It may also be downloaded from www.psych.mcgill.ca/abs/ cul/charles.html. HFN.m generates and presents an HFN stimulus according to a number of user-entered parameters that are described in detail below. It is designed for use with MATLAB 5.2 for Macintosh. It requires the Image Processing Toolbox (The Mathworks Inc., Natick, MA, www. mathworks.com) and the Psychophysics Toolbox ${ }^{1}$ (Brainard, 1997; Pelli, 1997; available free at psychotoolbox.org) to run.

\section{The HFN Stimulus}

The HFN stimulus is illustrated in Figure 1. It consists of a two-color random checker field overlaid with scattered black dots. For the purposes of this description, the checker colors are assumed to be red and green, and the dots black, but they can, in principle, be any color. The field scrolls horizontally and, at the same time, the colors of the checkers alternate. That is, all the red checkers become green and all the green ones become red several times per second (the rate of alternation can be varied; see the next section). The black dots do not change color or shape but simply move with the field.

When the two checker colors in an HFN stimulus are nonisoluminant, the alternation of the colors in combination with the movement of the stimulus produces a reversephi effect, so that the checker field appears to be drifting in the opposite direction to its actual movement. This occurs because the alternations in the checker colors are timed with their lateral movement, so that one never observes a same-colored checker to be exhibiting apparent motion in the veridical direction of stimulus movement (i.e., every time a square moves over, it changes to the opposite color). Thus, there is zero correlated motion in the actual direction the stimulus is moving in. At the same time, it is possible to observe some random squares of the same color apparently moving in the direction opposite to the actual motion of the stimulus, producing a weak correlation in the direction opposite to the actual motion of the stimulus.

When the two checker colors in the stimulus are made to be isoluminant, they fuse into an intermediate hue and the alternation effects are minimized so that it no longer produces the reverse-phi effect. The movement of the black dots is thus the only motion signal remaining, and the stimulus appears very robustly to move in the correct direction. The script described in the next section presents a version of the HFN stimulus in which one checker color is held at a constant radiance and the other is varied over time. When this is done, one gets the impression of a reversal of direction in the field, even though it always actually moves in one direction. The field's motion produces OKN eye movements that track the apparent direction of motion.

The appearance of the stimulus can vary along a number of dimensions, but we have found that a certain set of parameters is optimal for producing the effects described above. For instance, the speed of movement and the rate of checker alternation can be varied, but values of about $15 \% \mathrm{sec}$ and $15 \mathrm{~Hz}$, respectively, seem to produce the best reversephi and flicker fusion effects. Other parameters of the stimulus, such as checker size, dot size, and so on, can be varied more extensively without reducing the relevant effects. The MATLAB script described in the next section is designed to run by default with an optimal set of parameters.

\section{Using HFN.m}

To use HFN.m, place the function in the MATLAB folder (or a folder in MATLAB's path). Then, at the MATLAB prompt, type a command in the form:

HFN('paramter1', value1, 'parameter2', value2, . .);

There are 16 allowed input parameters. The user can provide values for as many or as few of these as desired, and they may be entered in any order, though the value must always immediately follow its associated parameter. Those for which no input value is given will simply remain at the default value. If no input values are provided at the command line, a dialog box will appear where these values can be entered. The values control such things as the colors of the checkers, the speed of the field's motion, and so on. The use of each parameter is detailed below. The first 10 parameters discussed control the size and color of various stimulus elements (the checkers, dots, and background); the remaining parameters control the timing and motion of the stimulus.

Note that the downloadable version of the script contains help text with similar information to what follows. This can be accessed by typing

$$
\text { help HFN }
$$

at the MATLAB prompt.

CheckSize: This variable controls the size of the individual checkers in the field. It is a two-element vector containing the horizontal and vertical dimensions of the checkers, measured in pixels. The default value is [20 20].

CheckNum: This gives the dimensions of the stimulus field. It is a two-element vector containing the number of checkers in the stimulus horizontally and vertically. The overall size of the HFN stimulus in pixels can be calculated as CheckSize(1)*CheckNum(1) horizontally and 
a ) Frame 1:0 $\mathrm{m} \mathrm{sec}$

Fra m e $2: 83 \mathrm{~m} \mathrm{sec}$

Frame $3: 167 \mathrm{~m} \mathrm{sec}$
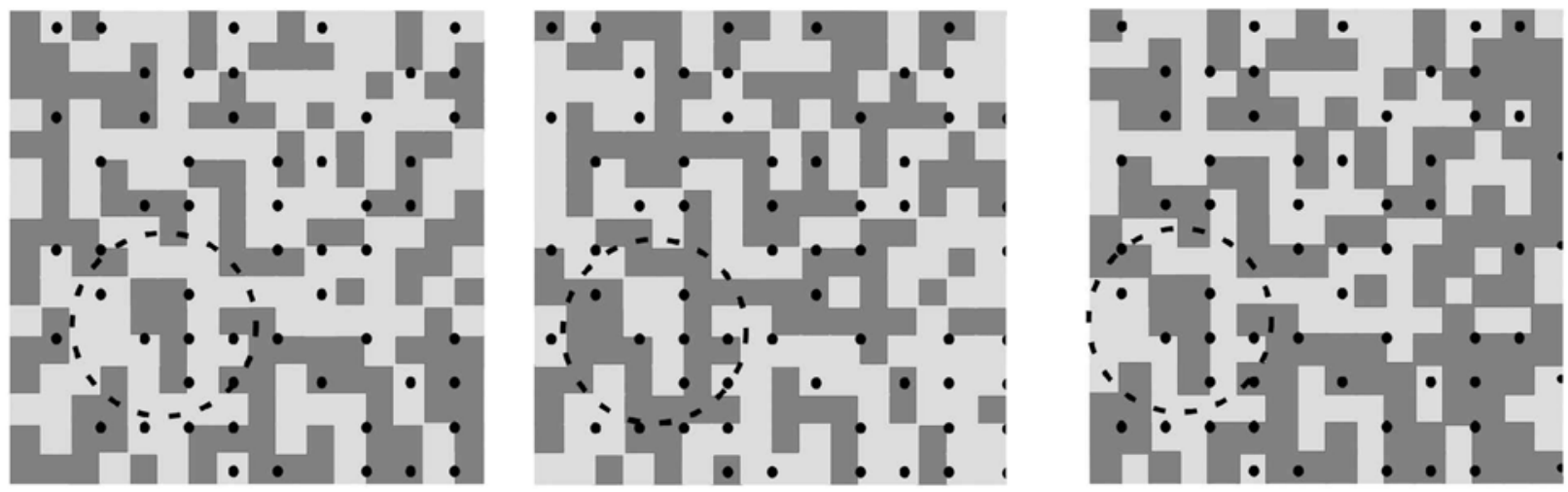

True direction of motion

b )
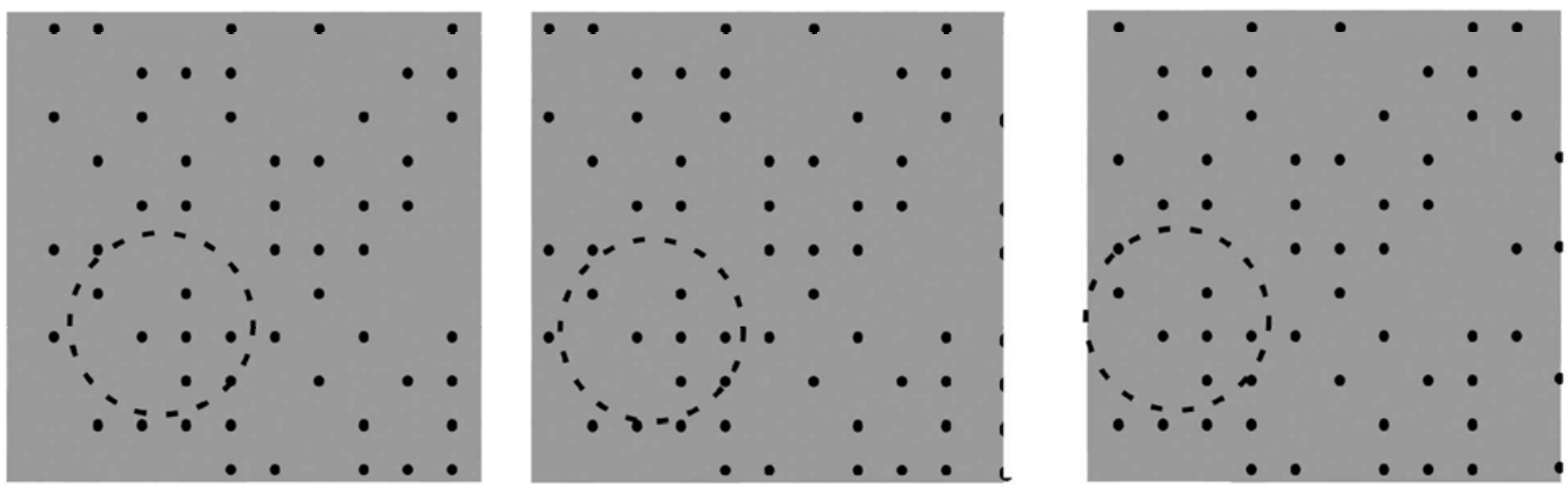

A p parentdirection of motion

Truedirection of motion

Figure 1. An illustration of the heterochromatic fusion nystagmus (HFN) stimulus. (a) When the HFN stimulus is not isoluminant, there are two competing motion signals, one of which produces an impression of motion in the direction opposite to that in which the stimulus is actually moving. With optimal stimulus parameters, this signal dominates, and the entire figure seems to drift in a direction opposite to its true direction. (b) When the HFN stimulus is isoluminant, the impression of nonveridical motion disappears, and the stimulus appears very robustly to move in the direction in which it is actually moving.

CheckSize(2)*CheckNum(2) vertically. The default value is [20 20], which yields a $400 \times 400$ pixel HFN stimulus if the default value of CheckSize is used.

ConstantRGB: The HFN.m script presents a version of the HFN stimulus in which one checker color is held constant while the other varies over time. This parameter controls the color of the constant checkers. It is an RGB triplet in the range $0-255$. The default value is [ $\left.\begin{array}{lll}0 & 128 & 0\end{array}\right]$, producing green.

VarRGBFile: This variable controls the range of colors through which the variable checkers are put. It is the name of a .mat file from which a color look-up table (CLUT) is loaded into the program. The value must be a string. If an empty string is entered, the program puts the checkers through a basic array of gray levels [0:255, 0:255, 0:255]. The default value is an empty string.
To create a file for input into this parameter, it is simply a matter of constructing a CLUT, assigning it to the variable name "VarRGB" and then saving that variable to a .mat file. For instance, if one wanted the variable checkers to be red, the following sequence of commands could be used to create a file that would achieve that result:

$$
\begin{aligned}
& \operatorname{VarRGB}=[0: 255 ; \operatorname{zeros}(1,256) ; \operatorname{zeros}(1,256)] \text {; } \\
& \text { save MyRedCLUT.mat VarRGB; }
\end{aligned}
$$

The file could then be called into the HFN script like this:

$$
\text { HFN('VarRGBFile', 'MyRedCLUT.mat'); }
$$

The above is just a simple example. A more sophisticated use of the VarRGBFile parameter would be to load a CLUT that provided for a linear increase in luminance. The contents of this file would vary on the basis of individual 
monitor characteristics. For information on creating such a linear CLUT, the reader is referred to Metha, Vingrys, and Badcock (1993) and Olds, Cowan, and Jolic ur (1999).

VarStartDex: This is a row index into the CLUT of the variable checkers (loaded via VarRGBFile) indicating which RGB triplet should be the starting color. The value must be a positive integer no larger than the number of rows in the CLUT. The default value is 200 . In the case of the default array of grays, this means the variable checkers start at a light gray.

DotSize: This is the size of the scattered black dots. It is a two-element vector containing the horizontal and vertical dimensions of the dots in pixels. The values must be positive integers. The default is [3 3].

DotSpacing: This determines the size of the grid on which the black dots appear. Each dot has a chance of appearing at any given point on the grid equal to DotDense (see below). The value must be a positive integer. The default value is 30 .

DotDense: This is the percentage probability of a dot's appearing at each point on the grid of possible dot locations (see DotSpacing above). The value may be any number from 0 to 100 . The default is 50 , meaning dots will appear at roughly half the possible locations.

DotRGB: This is an RGB triplet in the range 0-255, giving the color of the overlaid dots. The default is [ $\left[\begin{array}{lll}0 & 0 & 0\end{array}\right]$ (black).

BackRGB: An RGB triplet in the range 0 to 255 , giving the color of the screen background surrounding the HFN stimulus. The default is [255 255255 ] (white).

ShowTime: This is the amount of time (in seconds) that the stimulus is shown. Decimal seconds are allowed, but the script is not designed to produce high accuracy in terms of presentation time. The default is 20 .

MoveStep: This is the distance in pixels that the stimulus strip scrolls horizontally each time it is moved. Positive values produce leftward motion, negative values rightward motion. The value must be an integer. The default is 15 .

StripLength: The HFN stimulus is generated as a long strip across which a copying area is panned in order to produce a scrolling motion. This variable determines the overall length of the strip in multiples of the width of the on-screen stimulus. Thus, it determines how long the stimulus is shown before it repeats. Smooth wrap around is achieved by pasting the starting screen-full of stimulus to the end of the strip, so the overall length of the stimulus strip is (StripLength+1)* (CheckNum(1)*CheckSize(1)). The value must be a positive integer. The default is 2 , yielding a stimulus strip that is 1,200 pixels in length if the defaults for CheckSize and CheckNum are in place.

WaitFrames: This is the number of screen refreshes that are allowed to pass before the stimulus is scrolled (by MoveStep pixels) and the checker colors are reversed. The larger this value, the slower the scroll speed. For a good flicker fusion effect, this should be set to $\mathrm{H} / 30$, where $\mathrm{H}$ is the refresh rate of the screen in hertz. The value must be an integer and may not be less than 1 . The default value is 2 , appropriate for a $60-\mathrm{Hz}$ screen.
The speed at which an HFN stimulus scrolls depends on the refresh rate of the monitor, its dot pitch, and the values of the parameters MoveStep and WaitFrames. The speed in pixels per second is given by $(\mathrm{H} /($ WaitFrames $)) *$ MoveStep, where $\mathrm{H}$ is the refresh rate of the monitor in hertz. With the default values in place ( 2 for WaitFrames, 15 for MoveStep), a $60-\mathrm{Hz}$ monitor will produce a rate of speed of about 450 pixels per second. Given a dot pitch of 27 , this yields $450 / 27=16.67 \mathrm{~cm} / \mathrm{sec}$. Given a viewing distance of $57 \mathrm{~cm}$, this is about $16.67 \% \mathrm{sec}$, close to the optimal. For a $100-\mathrm{Hz}$ monitor, approximately the same rate can be achieved by increasing WaitFrames to 3 .

Note that HFN.m is not designed to produce perfect timing accuracy for the frames. Because of interrupt calls to the operating system, frames will occasionally be missed. Another version of the script, HFNrush.m, takes advantage of the Rush command from the Psychophysics Toolbox to produce better timing. Rush prevents some MacOS deferred tasks from interrupting MATLAB scripts, which otherwise might produce small but significant variations in the timing of a script's execution. HFNrush.m is similar to HFN.m in all ways except for the use of Rush. We have included HFN. $m$ here because the code is easier to understand and edit than is the code for HFNrush.m. Both are available at www.psych.mcgill.ca/labs/cvl/charle.html.

IncDelay: This is the number of times the stimulus moves (by MoveStep pixels) before the color of the variable checkers is incremented. As this value increases, the rate of change of color in the variable checkers decreases. The value must be a positive integer. The default value is 2 .

IncAmount: This is the amount by which the row index into the CLUT of the variable checkers is increased every IncDelay movement of the stimulus. The value must be a positive integer. The default is 3 .

The rate at which the luminance of variable checkers changes depends on the values in the CLUT for the variable checkers (loaded via VarRGBFile), the refresh rate of the monitor, and the values of parameters WaitFrames, IncDelay, and IncAmount. The rate in terms of CLUT entries per second is given by IncAmount/(WaitFrames * IncDelay* $(1 / \mathrm{H}))$, where $\mathrm{H}$ is the refresh rate of the monitor in hertz. With the default values in place, this yields a rate of about 7.5 entries per second on a $60-\mathrm{Hz}$ monitor. If, for instance, the values loaded via VarRGBFile were designed to represent equal steps of $0.3 \mathrm{~cd} / \mathrm{m}^{2}$ in luminance, this would mean an average increase of about $2.25 \mathrm{~cd} / \mathrm{m}^{2} / \mathrm{sec}$. Note that with the default behavior of the program (in which the whole gamut of gray levels are used), the luminance increase will not be constant for most monitors. This is due to the fact that for the vast majority of monitors, the relationship between RGB values and luminance is nonlinear and instead follows a gamma function. For more information on monitor nonlinearity and related issues, the reader is referred to Metha et al. (1993) and Olds et al. (1999).

\section{Examples}

The following are some examples of value settings that might be passed to HFN.m and the results. 


\section{HFN}

Simply entering the command without any passed parameters will cause a dialog window to be activated. The user may enter values for all the different parameters here. To simply hit the okay button without modifying any parameters will cause the function to display an HFN stimulus following the default parameters. The constant checkers will be green, the variable checkers will run through a gamut of gray levels, the checkers will be $20 \times 20$ pixels in size, and so on.

\section{HFN('CheckNum', [30 30], 'Constant RGB',} [0 128 128]);

This will produce an HFN pattern that is $600 \times 600$ pixels in size (i.e., $30 \times 30$ checkers, each $20 \times 20$ pixels), in which the constant checkers are a dark cyan color.

$\operatorname{VarRGB}=\operatorname{hsv}(64) * 255 ;$

save MyRainbow.mat VarRGB

HFN('VarRGBFile', 'MyRainbow.mat', 'VarStartDex', 32)

This sequence of commands produces a CLUT, saves it to a file, and then loads it into HFN.m. The CLUT here is taken from one of MATLAB's built-in colormap functions, $h s v . m$. The variable checkers will run through a wide range of colors. Note that in this case, the CLUT is only 64 entries long, so the starting index has to be reduced to fit into this range (to 32 , in this case).

\section{Conclusion}

The HFN stimulus can provide a useful means for equating the luminance of pairs of colors, especially with nonverbal subjects, such as animals and children. However, it is a complex stimulus, and it is difficult to program. HFN.m serves to demonstrate the stimulus and provides a guide as to how to program it. It is our hope that this will prove useful to other researchers and allow a broader application of the technique.

\section{REFERENCES}

Brainard, D. H. (1997). The Psychophysics Toolbox. Spatial Vision, 10, 433-436.

Cavanagh, P., Anstis, S., \& Mather, G. (1984). Screening for color blindness using optokinetic nystagmus. Investigative Ophthalmology \& Visual Science, 25, 463-466.

Cavanagh, P., Tyler, C. W., \& Favreau, O. E. (1984). Perceived velocity of moving chromatic gratings. Journal of the Optical Society of America A, 1, 893-899.

Chaudhuri, A., \& Albright, T. D. (1990). A new technique for estimating chromatic isoluminance in humans and monkeys. Visual Neuroscience, 5, 605-608.

Chaudhuri, A., \& Albright, T. D. (1992). Heterochromatic fusion nystagmus: Its use in estimating chromatic equiluminance in humans and monkeys. Vision Research, 32, 1745-1759.

Collin, C. A., \& Chaudhuri, A. (1996). A maximum motion method for detection of red-green color deficiency [Abstract]. Investigative Ophthalmology \& Visual Science, 37, 1062.

Collin, C. A., \& Chaudhuri, A. (1998). A maximum motion technique for assessment of color vision defects. Perception \& Psychophysics, 60, 1337-1343.

Cornelissen, F. W., Peters, E. M., \& Palmer, J. (2002). The Eyelink Toolbox: Eye tracking with MATLAB and the Psychophysics Toolbox. Behavior Research Methods, Instruments, \& Computers, 34, 613-617.

KAISER, P. K. (1996). Measuring spectral sensitivity. In The joy of visual perception: A Web book (chapter or section identifier). Available at http://www.yorku.ca/eye/brightne.htm.

Metha, A. B., Vingrys, A. J., \& BADCock, D. R. (1993). Calibration of a color monitor for visual psychophysics. Behavior Research Methods, Instruments. \& Computers, 25, 371-383.

Olds, E. S., CowAn, W. B., \& Jolicceur, P. (1999). Effective color CRT calibration techniques for perception research. Journal of the Optical Society of America A, 16, 1501-1505.

Pelli,D. G. (1997). The video toolbox software for visual psychophysics: Transforming numbers into movies. Spatial Vision, 10, 437-442.

\section{NOTE}

1. A version of the Psychophysics Toolbox is available for MATLAB for Windows platforms, but it is still in the testing stage (see psychtoolbox.org/win.html for more information). Once this version of the toolbox is more fully developed, HFN.m should run with it, but we have not yet tested it on that platform. Once the platform is fully available for the PC, we will test it and update our scripts on the Web site. 
APPENDIX

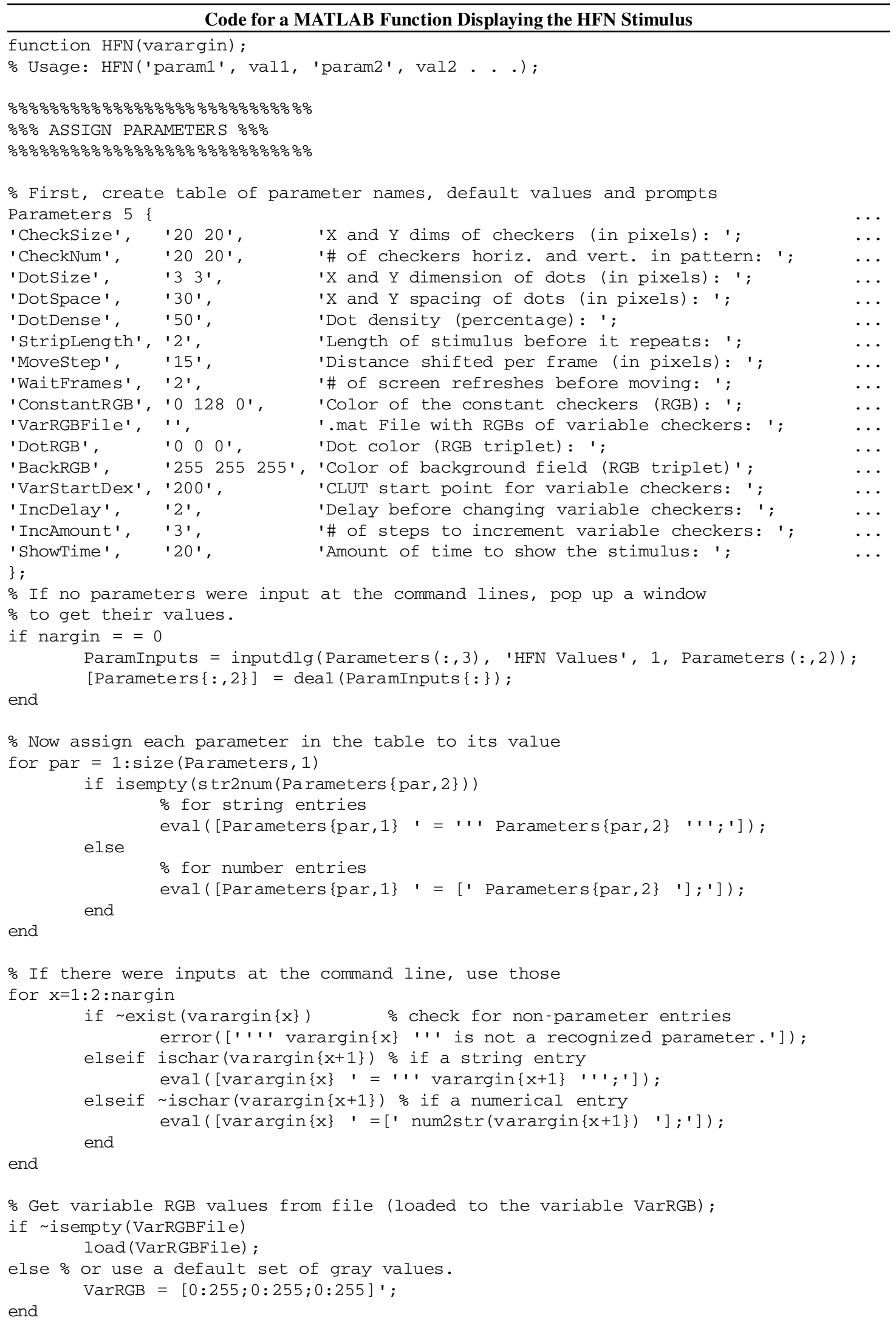




\section{APPENDIX (Continued)}

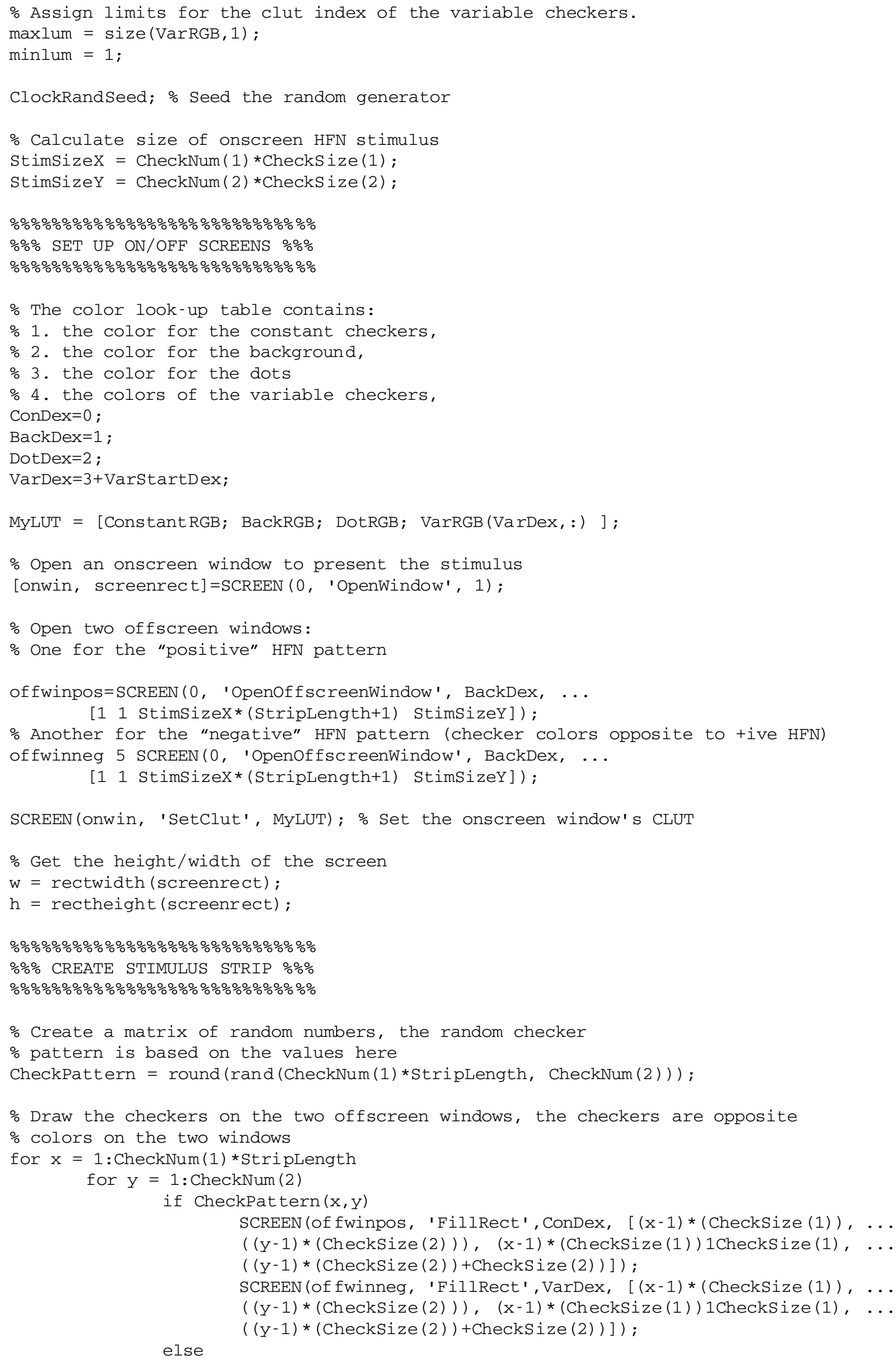




\section{APPENDIX (Continued)}

SCREEN (of fwinpos, 'FillRect', VarDex, [(x-1)*(CheckSize (1)), ... $((y-1) *($ CheckSize $(2))),(x-1) *($ CheckSize $(1)) 1$ CheckSize $(1), \ldots$ $((\mathrm{y}-1) *($ CheckSize (2)) +CheckSize (2)) ]) ; SCREEN (of fwinneg, 'FillRect', ConDex, [(x-1)*(CheckSize (1)), ... $((y-1) *($ CheckSize $(2))),(x-1) *($ CheckSize $(1))$ 1CheckSize $(1), \ldots$ $((y-1) *($ CheckSize (2)) +CheckSize (2)) ]) ;

end

end

end

$\%$ Get the dimensions of the offscreen patterns.

posrect = SCREEN (offwinpos, 'rect');

negrect = SCREEN (of fwinneg, 'rect');

$\%$ Draw the dots on both patterns

for $\mathrm{x}=1$ : (StimSizeX* (StripLength+1)) /DotSpace

for $y=1:$ StimSizeY/DotSpace

if randi $(100)<=$ DotDense

SCREEN (of fwinpos, 'FillRect', DotDex,

$\left[x^{*}(\operatorname{DotSize}(1))+(\operatorname{Dot}\right.$ Space* $(x-1))$,

$\mathrm{y}^{*}(\operatorname{DotSize}(2))+($ DotSpace* $(\mathrm{y}-1))$

$\mathrm{x}^{*}(\operatorname{DotSize}(1))+\operatorname{DotSize}(1)+(\operatorname{Dot}$ Space*(x-1)),

$\left.\left.\mathrm{y}^{*}(\operatorname{DotSize}(2))+\operatorname{DotSize}(2)+(\operatorname{DotSpace}(\mathrm{y}-1))\right]\right)$;

SCREEN (of fwinneg, 'FillRect', DotDex,

$\left[\mathrm{x}^{*}(\operatorname{DotSize}(1))+(\operatorname{Dot}\right.$ Space $*(\mathrm{x}-1))$

$\mathrm{Y}^{*}(\operatorname{DotSize}(2))+($ DotSpace* $(\mathrm{y}-1))$,

$x^{*}(\operatorname{DotSize}(1))+\operatorname{DotSize}(1)+(\operatorname{Dot}$ Space* $(x-1))$,

end

$\left.\left.\mathrm{y}^{*}(\operatorname{DotSize}(2))+\operatorname{Dot} \operatorname{Size}(2)+(\operatorname{Dot} \operatorname{Space} *(\mathrm{y}-1))\right]\right)$;

end

$\%$ Copy the start of one pattern onto the end of the other so that the pattern \% won't "skip" when it scrolls around SCREEN('CopyWindow', offwinneg, offwinneg, [1 1 stimSizeX StimSizeY]-1,... [posrect(3) - (StimSizeX-1) 0 posrect(3) StimSizeY-1]); SCREEN('CopyWindow', offwinpos, offwinpos, [1 1 stimsizeX StimSizeY]-1,... [negrect(3)-(StimSizeX-1) 0 negrect(3) StimSizeY-1]);

$\% \% \% \% \% \% \% \% \% \% \% \% \% \% \% \% \% \% \% \% \%$

\% $\%$ PRESENT STIMULUS \% $\%$

$\% \% \% \% \% \% \% \% \% \% \%$

\% Start position of the copying area that pans across the offscreen patterns. NowPos $=0$;

lum = VarStartDex; $\quad \%$ The index of the RGB value for the variable checkers

FlushEvents('keyDown'); \% Make sure no keypresses are pending

tstart $=$ getsecs; $\quad \%$ Get starting time

iters=1; $\quad \%$ Number of steps the stimulus has moved through

flushevents ('keyDown')

while 1; $\%$ Go until time limit reached, then break out.

$$
\begin{aligned}
& \text { if CharAvail } \\
& \text { break; } \\
& \text { end }
\end{aligned}
$$


APPENDIX (Continued)

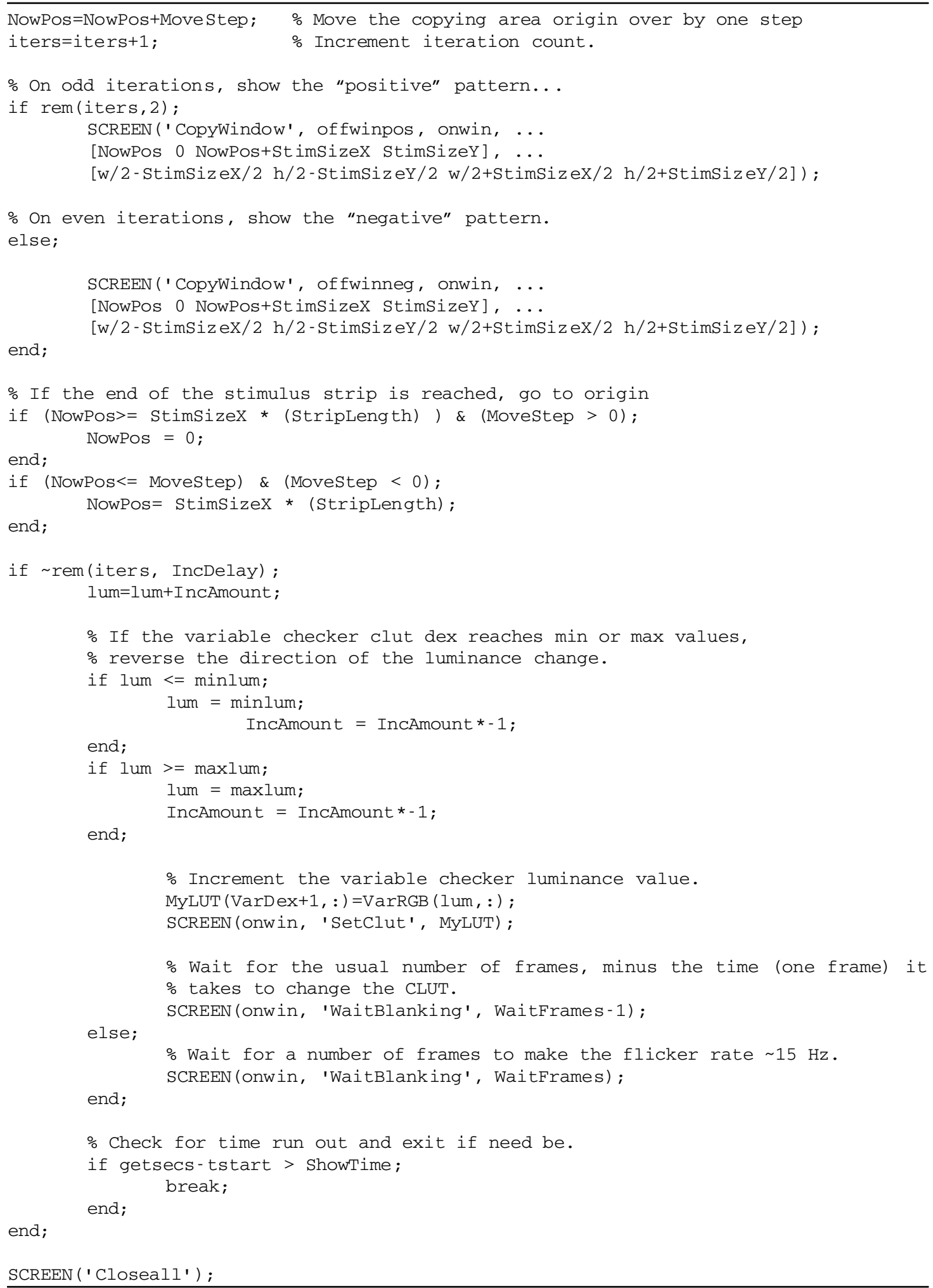

(Manuscript received March 27, 2002;

revision accepted for publication July 15,2002 .) 